\title{
GALACTOSEMIA CLÁSICA (A PROPÓSITO DE UN CASO) UNIDAD ESTUDIOS ESPECIALES ADJUNTO CENISMI, HOSPITAL INFANTIL DR. ROBERT REID CABRAL (HIRRC), REPÚBLICA DOMINICANA
}

\section{CLASSICAL GALACTOSEMIA (in relation to a case) Special Studies Unit attached CENISMI, Dr. Robert Reid Cabral Children's Hospital (HIRRC), Dominican Republic}

\author{
Mayra Fortuna Pérez*, Félix Díaz Rancaño**, \\ Ceila Pérez de Ferrán**, Whous Kendy De Aza***
}

Recibido: 8/8/2017 Aprobado: 5/9/2017

DOI: $X X X X X X$

\section{Resumen}

La galactosemia es una ocurrencia rara debido a la falla del metabolismo del azúcar galactosa en la ruta de Leloir, usada por el cuerpo para transformar D-galactosa a D-glucosa 1 fosfato, para lo cual requiere la participación de las enzimas GALK1, GALT y GALE. El gen que codifica la enzima galactosa 1 fosfato uridil transferasa (GaLT) se localiza en el cromosoma 9p13. Es la primera enzima identificada en este trastorno y también es la causa más común de galactosemia tipo I y/o galactosemia clásica (OMIM No. 230400).

En este trabajo presentamos el caso de un niño de un año y tres meses de edad que, después de tres meses de vida, fue hospitalizado en varias ocasiones a causa de infecciones del tracto urinario y neumonía.

$\mathrm{Al}$ año de edad presentó anemia severa, desnutrición marasmática, retraso motor, opacidad del cristalino bilateral y hepatomegalia de $7 \mathrm{~cm}$ por debajo del reborde costal derecho.

Palabras clave: galactosemia tipo I; deficiencia de galactosa 1 fosfato uridil transferasa; cataratas.

\footnotetext{
* Jefa del Servicio del Hospital Robert Read Cabral, República Dominicana. Mayrafortuna@gmail.con

** Médicos ayudantes

*** Médico general
}

\section{Summary}

Galactosemia is a rare occurrence due to the failure of the sugar metabolism galactose in the Leloir pathway, a route used by the body to transform $\mathrm{D}$-galactose to $\mathrm{D}$-glucose 1 phosphate, requiring the participation of the enzymes GALK1, GALT and GALE. The gene encoding the galactose 1 phosphate uridyl transferase (GaLT) enzyme is located on chromosome 9p13. It is the first enzyme identified in this disorder and it is also the most common cause of galactosemia type I and/or classic galactosemia (OMIM No. 230400).

We present a case of a one-year-old male child who, after three months of life, was hospitalized repeatedly for urinary tract infections and pneumonia.

At one year of age, he presented severe anemia, marasmic malnutrition, motor retardation, opacity of the bilateral lens and hepatomegaly $7 \mathrm{~cm}$ below the right costal ridge.

Keywords: Galactosemia type I; deficiency of galactosa 1 fosfato uridil transferasa; catarats.

\section{Introducción}

La galactosemia es una enfermedad originada por el fallo del metabolismo del azúcar galactosa en la ruta de Leloir, considerada para la transformación de D-galactosa a D-glucosa 1 fosfato, requiriendo 
la participación de las enzimas GALK1, GALT Y GALE $^{1,2}$.

La galactosemia tipo I o clásica es una enfermedad autosómica recesiva, causada por la mutación del gen localizado en el cromosoma 9 p13, encargado de la codificación de la enzima GALT, que actúa en la galactosa 1 fosfato, transformándola en glucosa 1 fosfato para continuar la vía de metabolización de este azúcar. La deficiencia o ausencia total de esta enzima por mutación del gen acarrea trastornos multisistémicos en el aparato digestivo, los rińones, el sistema nervioso central y los ovarios.

El gen que codifica la enzima galactosa 1 fosfato uridil transferasa (GALT) fue el primero que se identificó y es el causante de la enfermedad galactosemia tipo I y/o galactosemia clásica (OMIM No. 230400). Esta enfermedad se presenta en $1 / 40.000$ a 1/60,000 recién nacidos. Cabe destacar que en Irlanda presenta una frecuencia mucho menor $(1 / 16,000)^{3}$.

Existen más de 230 tipos de mutaciones a nivel mundial, siendo la más común la Q188R, seguida de S135L, K285N y la variante N314D. La galactosemia clásica $(\mathrm{G})$ presenta heterogeneidad genética diversa, así tenemos galactosemia homocigota (G/G, la que posee actividad enzimática menor de un $5 \%$; variante Duarte, con diversidades tales como variantes $\mathrm{D} / \mathrm{D}, \mathrm{D} / \mathrm{G}$, en la que la actividad enzimática varía entre un 25 y un $75 \%$ y, por lo tanto, estos niños no presentan afectación clínica ni orgánica, y solo es detectable en el tamizaje neonatal ${ }^{4}$.

La sepsis por E. Coli se presenta en un 10-50\% de los pacientes con esta enfermedad, aunque además pueden ser infectados por Klebsiela, Enterobacter, Streptococus faecalis, entre otros, debido a la disminución de la actividad bactericida de los leucocitos $^{5}$. Estos pacientes suelen presentar cataratas en un $30 \%$ de los casos, por acumulación del galactitol en el cristalino.
En el sistema digestivo el órgano más afectado es el hígado, causando alteraciones que van desde la insuficiencia hepática hasta la cirrosis. La afectación renal ocasiona el Síndrome de Fanconi, que conlleva a insuficiencia renal y alteraciones en el sistema nervioso central ${ }^{6}$ por acumulación de metabolitos anómalos, lo que acarrea déficit cognitivo de diferentes grados en el paciente.

El organismo con este trastorno metabólico utiliza la vía alterna, que se traduce con aumento de galactitol, galactonato y aumento de galactosa 1 fosfato; esta última se acumula en el organismo y actúa unida al galactitol, lo que ocasiona daños en los órganos dianas ${ }^{7,8}$.

La galactosemia clásica en el período neonatal es potencialmente mortal, presentando un cuadro de insuficiencia hepática, con evidencia de ictericia, alteraciones del tiempo de coagulación y cirrosis hepática; a nivel renal se producen lesiones importantes, tales como el Síndrome de Fanconi y el raquitismo hipofosfatémico. Las niñas, en gran porcentaje de casos, presentan disfunción gonadal, siendo usual la amenorrea y la infertilidad.

El diagnóstico de la galactosemia clásica se basa en la determinación de la concentración de galactosa-1-fosfato en eritrocitos, que será $>10 \mathrm{mg} / \mathrm{dl}$ y la actividad enzimática GALT en el eritrocito estará ausente o $\leq 10 \%$ de la actividad de control. En estos pacientes se restringen todos los productos lácteos, algunas frutas y algunos vegetales, así como los medicamentos que posean galactosa como conservante.

El diagnóstico diferencial con la galactosemia tipo II se evidencia por la deficiencia de la enzima galactokinasa (GALK) y la presencia de cataratas por acumulación del galactitol en el cristalino, sin alteraciones hepáticas, presentando aumento de galactosa y aumento de la excreción de galactitol, sin aumento de galactosa 1 fosfato, ya que la actividad enzimática de la GALT en el eritrocito es normal. 
Otro diagnóstico diferencial es con la galactosemia tipo III, producida por la deficiencia de la enzima UDP-galactosa 4-epimerasa (GALE) ) en forma severa, presentando manifestaciones clínicas indistinguibles, con alteraciones hepáticas y cataratas, diferenciándose de la galactosemia clásica por el aumento de las concentraciones de galactosa-1-fosfato en eritrocitos, elevada con actividad enzimática de la GALT normal, distinguiéndose solo por la cuantificación enzimática y/o la identificación del gen. La detección de la deficiencia de GALE (galactosa 4 epimerasa) es diagnóstica.

Cuando existan trastornos hepáticos y renales, debemos tener en cuenta en el diagnóstico diferencial la Tirosinemia y la Fructosemia.

\section{Reporte del caso}

Lactante masculino de un año y tres meses de edad, hijo de madre de 27 años, padres no consanguíneos, G1 P0 C1; cursó con chequeos prenatales regulares. Producto único, llanto al nacer, peso de 7 libras. Presentó ictericia a los cuatro días de vida, lo que ameritó fototerapia. A partir de los tres meses, se iniciaron infecciones de las vías urinarias y neumonías a repetición.

Fue ingresado al hospital al año de edad por presentar anemia severa, desnutrición tipo marasmática, retraso motor, opacidad del cristalino bilateral y hepatomegalia de $7 \mathrm{~cm}$ por debajo del reborde costal derecho.

Se realizaron estudios bioquímicos que revelaron elevaciones de las transaminasas, fosfatasa alcalina, tiempo de protrombina, hiperbilirrubinemia bifásica e hipoglucemia; GG TP normal, HIV, HBsAg y HVC negativos.

El TAC craneal reportó amplia hipodensidad en sustancia blanca a nivel bifrontal, corona radiada bitemporal y biparietal. La sonografía abdominal evidenció hepatomegalia con presencia de líquido ascítico.

Frente a la historia de infecciones recurrentes de las vías urinarias y sepsis a E. Coli, cataratas bilaterales, afección hepática con hepatomegalia y alteraciones bioquímicas hepáticas, se consideró un trastorno metabólico relacionado a la galactosa, probable galactosemia clásica, por lo que se procedió a la toma de muestra de orina para detectar la sustancia reductora (Benedict), reportándose positiva $(++++)$ en ausencia de glucosa. No se realizó cuantificación de galactosa en sangre, galactitol y galactosa 1 fosfato, por su alto costo.

Se eliminó la ingesta de leche de vaca u otro animal y sus derivados, también algunas frutas, y se introdujo la leche de soya y el calcio.

$\mathrm{Al}$ mes de iniciar la restricción dietética, presenta avances en su desarrollo motor (se sentó solo) y seis meses más tarde desapareció la hepatomegalia, las pruebas hepáticas se normalizaron y disminuyeron también los procesos infecciosos.

El paciente se ausentó, reapareciendo a los cuatro años y cinco meses. Se observó únicamente déficit cognitivo (no hace frases largas); el examen físico reveló buen desarrollo pondo-estatural, sin evidencia de hepatomegalia.

Se cuantificó la enzima Galactosa-1-FosfatoUridiltransferasa en Mayo Clinic LaboratoriesRochester USA, reportándose ausencia de la actividad enzimática $(0.0 \mathrm{nmol})$, lo que confirmó el diagnóstico de galactosemia tipo I.

\section{Discusión}

La galactosemia clásica es una enfermedad autosómica recesiva causada por la mutación del gen (GALT), encargado de la codificación de la enzima galactosa-1 fosfato uridil transferasa, que actúa en la galactosa 1 fosfato, transformándola en glucosa 
1 fosfato para continuar la vía de metabolización de este azúcar; su deficiencia o ausencia total por mutación del gen acarrea trastornos multisistémicos en el sistema digestivo, los riñones, el sistema nervioso central y los ovarios.

El paciente estudiado presentó múltiples manifestaciones, tales como retraso psicomotor, desnutrición importante, cataratas bilaterales e historia de infecciones recurrentes de las vías urinarias, sepsis por E. Coli, neumonía a repetición y afectación hepática con aumento de las transaminasas, bilirrubina, tiempo de coagulación y disminución de proteínas, lo que nos hizo sospechar de un trastorno metabólico ligado a la galactosa, aumentando la sospecha al evidenciarse en el examen de orina la sustancia reductora (Benedict positiva) con glucosa negativa, por lo que se procedió a la suspensión de la leche y sus derivados, observándose en el paciente una mejoría importante y una reversión del cuadro hepático.

La imposibilidad de la cuantificación de galactosa, galactonato y galactosa 1 fosfato ${ }^{9,10}$, por los altos costos que conllevaba, nos obligó a realizar exclusivamente la cuantificación de la enzima galactosa 1 fosfato uridil transferasa, realizado en Mayo Clinic Laboratories-Rochester Main Campus, que reportó la ausencia total de esta enzima.

En nuestro país, la detección temprana de algunos errores innatos del metabolismo está por iniciarse. Al principio, las enfermedades a tamizar serán fenilcetonuria, galactosemia, hipotiroidismo congénito, hemoglobinopatías y deficiencia de glucosa 6 fosfato deshidrogenasa. Esperamos que posteriormente se amplíe la cobertura para la detección de otros errores innatos del metabolismo.

\section{Bibliografía}

1. McCorvie TJ, Timson DJ. The Structural and Molecular Biology of Type I Galactosemia:
Enzymology of Galactose 1-phosphate Uridylyltransferase. IUBMB Life. 2011; 63(9): 694-700.

2. McCorvie1 TJ, Kopec J, Pey AL, Fitzpatrick F, Patel D, Chalk R, Shrestha L, Yue WW. Molecular basis of classic galactosemia from the structure of human galactose 1-phosphate uridylyltransferase. Human Molecular Genetics. 2016; 25(11): 1-11.

3. Berry GT. Galactosemia: When is it a newborn screening emergency? Molecular Genetics and Metabolism. 2012; 106(1): 7-11.

4. Rathi N, Rathi A. Galactosemia Presenting as Recurrent Sepsis. Journal of Tropical Pediatrics. 2011; 57(6): 487-489.

5. Bosch AM. Classic galactosemia: Dietary dilemmas. J Inherit Metab Dis. 2011; 34 (2): 257-260.

6. Berry G. Is prenatal myo-inositol deficiency a mechanism of CNS injury in galactosemia? Journal of Inherited Metabolic Disease. 2011; 34(2): 345-355.

7. Berry G T. Classic Galactosemia and Clinical Variant Galactosemia, Synonyms: GALT Deficiency, Galactose-1-Phosphate Uridyltransferase Deficiency. GeneReviews [Internet]. 2014 (citado 7 agosto 2016); Disponible en: https://www.ncbi.nlm.nih.gov/ books/NBK1518/\#_galactosemia_Diagnosis_

8. Ficicioglu C, Hussa C, Gallagher PR, Thomas N, Yager C. Monitoring of Biochemical Status in Children with Duarte Galactosemia: Utility of Galactose, Galactitol, Galactonate, and Galactose 1-Phosphate. Clinical Chemistry. 2010; 56(7): 1177-1182.

9. Rubio-Agusti I, Carecchio M, Bhatia KP, Kojovic M, I Parees I, Chandrashekar HS, Footitt EJ, Burke D, Edwards MJ, Lachmann 
RH, Murphy E. Movement Disorders in Adult Patients With Classical Galactosemia. Movement Disorders. 2013; 28(6): 804-810.

10. McCorvie TJ, Timson DJ. Structural and Molecular Biology of Type I Galactosemia: Disease-associated Mutations. IUBMB Life. 2011; 63(11): 949-954. 